\title{
Cultural adaptation and validation of the Polish version of the Pulmonary Arterial Hypertension-Symptoms and Impact (PAH-SYMPACT) questionnaire
}

\author{
Kathie Sarzyńska1', Jacek Polański², Grzegorz Kopeć3 ${ }^{3}$ Ewa Mroczek4, Beata Jankowska-Polańska ${ }^{5}$ \\ 'Division of Nervous System Diseases, Department of Clinical Nursing, Faculty of Health Science, Wroclaw Medical University, Wrocław, Poland \\ 2Department of Internal Medicine, Occupational Diseases, Hypertension and Clinical Oncology, Wroclaw Medical University, Wrocław, Poland \\ ${ }^{3}$ Department of Cardiac and Vascular Diseases, Jagiellonian University Medical College, John Paul II Hospital in Krakow, Kraków, Poland \\ ${ }^{4}$ Area of Congenital Defects and Pulmonary Hypertension, Jan Mikulicz-Radecki University Teaching Hospital, Wrocław, Poland \\ ${ }^{5}$ Research and Innovation Center, $4^{\text {th }}$ Military Teaching Hospital, Wrocław, Poland
}

Correspondence to: Kathie Sarzyńska, MD, Department of Clinical Nursing, Wroclaw Medical University, Bartla 5, 51-618 Wrocław, Poland, phone: + 48883652438 , e-mail: kathie@vp.pl Copyright by the Author(s), 2021 Kardiol Pol. 2021; 79 (12): 1372-1374 DOI: 10.33963/KP.a2021.0149 Received: August 20, 2021 Revision accepted: October 28, 2021 Published online: October 30, 2021

\section{INTRODUCTION}

Pulmonary arterial hypertension $(\mathrm{PAH})$ is a disease that leads to the development of right ventricular failure and death despite optimal treatment [1]. A study published by Kopeć et al. [2, 3] in 2020 shows that the estimated prevalence of patients with $\mathrm{PAH}$ in Poland is 30.8 per million adults. Recent advances in therapy have improved symptoms severity, functional capacity and survival, but PAH still remains an incurable disease [4].

The main symptom of PAH is dyspnea. However, the experience of this, as well as other symptoms, is subjective, so there is a need to adapt specific tools to assess the severity of symptoms and their impact on patients' functioning. Recommendations for a PAH study endpoint now emphasize the importance of measuring patient-reported outcomes as a secondary endpoint in clinical trials $[5,6]$. In the few studies carried out so far in Poland among patients with $\mathrm{PAH}$, only generic tools have been used, i.e. general tools for quality-of-life assessment regardless of the type of disease entity [7], which may not reflect the symptoms and effects of PAH and their impact on quality of life (QoL). Due to the need for research in this group of patients, we have undertaken to translate and validate a tool that can be used to assess QoL and relate it to data presented by other centers in Europe and worldwide.

\section{METHODS}

\section{Questionnaires used in the psychometric validation process}

- The Cambridge Pulmonary Hypertension Outcome Review (CAMPHOR) questionnaire is a specific, standardized tool (Polish version) for assessing the quality of life in patients with pulmonary hypertension [8];

- WHO-QOL BREF — a standardized, abbreviated Polish version of the generic questionnaire - assessing the QoL of a patient with any disease entity [9];

- PAH-SYMPACT (Pulmonary Arterial Hypertension-Symptoms and Impact) is a specific questionnaire to assess the quality of life of patients with pulmonary arterial hypertension [5].

\section{Patients}

The study included 55 patients who met the inclusion criteria: $>18$ years, diagnosis of $\mathrm{PAH}$, entry into the national pulmonary hypertension database, written informed consent of the patient for participation in the study, health condition allowing participation in the study, and completion of questionnaires. All questionnaires were administered twice approximately 2-4 weeks apart.

\section{The process of cultural adaptation of the Polish version of the PAH-SYMPACT questionnaire}

Permission to translate and validate the English version of the PAH-SYMPACT ques- 
Table 1. Scores for each subscale of the PAH-SYMPACT questionnaire, Cronbach's alpha, and Spearman correlation coefficient

\begin{tabular}{l|c|c|c|c}
\hline $\begin{array}{c}\text { PAH-SYMPACT questionnaire } \\
\text { subscales }\end{array}$ & $\begin{array}{c}\text { Cronbach's alpha } \\
(\mathbf{n = 5 5 )}\end{array}$ & Median (IQR) & Min-max & $\begin{array}{c}\text { Spearman correlation } \\
\text { coefficient (n= 52) }\end{array}$ \\
$\begin{array}{l}\text { Cardiopulmonary symptoms } \\
\text { in Spearman correlation }\end{array}$ \\
Cardiovascular symptoms & 0.706 & $1.00(0.50-1.38)$ & $0.00-2.17$ & 0.945 \\
Physical effects & 0.806 & $0.40(0.20-0.85)$ & $0.00-2.0$ & 0.911 \\
Psychological effects & 0.904 & $1.14(0.54-2.0)$ & $0.14-3.86$ & 0.993 \\
\hline
\end{tabular}

Abbreviations: IQR, interquartile range; PAH-SYMPACT, Pulmonary Arterial Hypertension-Symptoms and Impact

tionnaire was obtained from the MAPI Research Gate. The procedure for the cultural adaptation of the Polish version of the PAH-SYMPACT questionnaire was conducted in 3 stages:

- Step 1: Translation process (Questionnaire forward translation, Questionnaire back translation);

- Step 2: Cognitive debriefing interviews;

- Step 3: Psychometric validation.

To determine the construct validity of the translated Polish version of the PAH-SYMPACT (PL) questionnaire, the reliability coefficient (Cronbach's alpha) for each subscale was calculated. Moreover, the internal consistency of the translated version using the test-retest method was determined.

\section{Statistical analysis}

The analysis of quantitative variables was conducted by calculating the mean, standard deviation, median, first and third quartiles, minimum, and maximum. The analysis of qualitative variables was performed by calculating the number and percentage of occurrences of each value. The internal consistency of the PAH-SYMPACT was assessed using Cronbach's alpha. Convergent validity was assessed by correlating all questionnaires. Correlations between test and retest scores were analyzed using Spearman's correlation coefficient. Thus, all $P$-values below 0.05 were interpreted as indicating significant correlations. The analysis was performed in the program $\mathrm{R}$, version 3.6.3 (R Development Core Team, Vienna, Austria).

\section{Ethical considerations}

The study was approved by University Bioethics Committee (approval number 538/2019). All patients provided informed consent. All study participants were informed that the study is conducted anonymously, and participation is voluntary.

\section{RESULTS AND DISCUSSION}

The average age (standard deviation [SD]) of respondents was 56.22 (17.25) years and more than half (69\%) were over 60 years. Approximately $85 \%$ of participants suffered from idiopathic $\mathrm{PAH}$, and the rest had $\mathrm{PAH}$-related connective tissue disorders.

The study shows that the Polish adaptation of PAH-SYMPACT was successful. Carefully developed QoL scales provide a holistic picture of the impact of disease and its treatment on patients. The new language version meets the expectations of good internal consistency, test-retest reliability, and convergent and known group validity. The resulting version reliably examines symptoms (resulting from the disease), as well as outcomes (consequences of symptoms) in patients with $\mathrm{PAH}$.

The median (interquartile range [IQR]) on the subscales: cardiopulmonary symptoms, cardiovascular symptoms, physical effects, and psychological effects was 1.0 (0.5$-1.38), 0.4(0.2-0.85), 1.14(0.54-2.0)$, and $0.75(0.5-1.25)$, respectively (Table 1 ).

The reliability for each subscale of the translated Polish version of the PAH-SYMPACT questionnaire was determined using Cronbach's alpha. In all 4 domains, all subscales were shown to be reliable. Cronbach's alpha coefficient for each subscale is above 0.7 , an alpha above 0.7 is assumed to be a reliable scale [10]. Removing any of the items does not improve the alpha coefficient (Table 1). Therefore, it can be concluded that the created scale is well constructed and does not need to be changed.

Consistency over time was examined by correlating the results of the first and second tests. The result indicated a very high consistency, between the repeated tests performed. Indeed, the correlation coefficient was always above 0.9 and was statistically significant $(P<0.05)$ for each subscale (Table 1). There were no cultural/linguistic differences between Poland and the creators'version in terms of what daily life is like for PAH patients [5]. Validation of the original American version of the PAH-SYMPACT questionnaire demonstrated Cronbach's alpha coefficient $>0.8$, and Spearman's correlation coefficient in the test-retest study $>0.7$ (Table 1). The Polish version of the PAH-SYMPACT questionnaire has a good design and is a reliable version of the questionnaire for assessing QoL. Moreover, each domain of the PAH-SYMPACT, correlated with all domains of the CAMPHOR questionnaire, which indicates that both tools can be used interchangeably or concurrently with success in the assessment of the QoL among patients with PAH. However, it should be emphasized that only the PAH-SYMPACT questionnaire is dedicated to a narrow group of PAH patients. An American cultural adaptation of the PAH-SYMPACT questionnaire [5] obtained similar results as the Polish version. Scores for each domain: cardiopulmonary symptoms, cardiovascular symptoms, physical effects, psychological effects: $1.0 ; 0.4 ; 1.3 ; 0.9$ correlated with our own results: $1.03 ; 0.56 ; 1.32 ; 0.89$, respectively. In both 
studies, the results should be interpreted as low severity of symptoms and effects. Only in the study performed by Chin et al. [5], in the subscale of cardiovascular symptoms, the result can be interpreted as the absence of symptoms. However, it should be emphasized that despite the similarities in both studies, such as female predominance in the study group $>60 \%$ and mean age $>55$ years, our study included only patients chronically treated for $\mathrm{PAH}$, in contrast to the study by Chin et al. [5] where the included patients were incidental.

\section{Limitations}

The study had some limitations. One of them was a relatively small number of participants, and the other was a single-center study.

\section{CONCLUSION}

Despite the limitations, the cultural adaptation of the Polish version of the PAH-SYMPACT questionnaire demonstrated that it provides a new accurate and reliable instrument for assessing health-related quality of life in Polish patients with PAH.

\section{Article information}

Conflict of interest: None declared.

Open access: This article is available in open access under Creative Common Attribution-Non-Commercial-No Derivatives 4.0 International (CC BY-NC-ND 4.0) license, allowing to download articles and share them with others as long as they credit the authors and the publisher, but without permission to change them in any way or use them commercially. For commercial use, please contact the journal office at kardiologiapolska@ptkardio.pl.

How to cite: Sarzyńska K, Polański J, Kopeć G, et al. Cultural adaptation and validation of the Polish version of the Pulmonary Arterial Hypertension-Symptoms and Impact (PAH-SYMPACT) questionnaire. Kardiol Pol. 2021; 79(12): 1372-1374, doi: 10.33963/KP.a2021.0149.

\section{REFERENCES}

1. Benza RL, Miller DP, Barst RJ, et al. An evaluation of long-term survival from time of diagnosis in pulmonary arterial hypertension from the REVEAL Registry. Chest. 2012; 142(2): 448-456, doi: 10.1378/chest.11-1460, indexed in Pubmed: 22281797.

2. Kopeć G, Kurzyna M, Mroczek E, et al. Characterization of patients with pulmonary arterial hypertension: data from the Polish Registry of Pulmonary Hypertension (BNP-PL). J Clin Med. 2020; 9(1): 173, doi: 10.3390/jcm9010173, indexed in Pubmed: 31936377.

3. Kopeć G, Kurzyna M, MroczekE, et al. Database of Pulmonary Hypertension in the Polish Population (BNPPL): design of the registry. Kardiol Pol. 2019; 77(10): 972-974, doi: 10.33963/KP.14988, indexed in Pubmed: 31553328.

4. Galie N, Humbert M, Vachiery JL, et al. 2015 ESC/ERS Guidelines for the diagnosis and treatment of pulmonary hypertension. Kardiol Pol. 2015; 73(12): 1127-1206, doi: 10.5603/KP.2015.0242, indexed in Pubmed: 26727670.

5. Chin KM, Gomberg-Maitland M, Channick RN, et al. Psychometric validation of the pulmonary arterial hypertension-symptoms and impact (PAH-SYMPACT) questionnaire: results of the SYMPHONY trial. Chest. 2018; 154(4): 848-861, doi: 10.1016/j.chest.2018.04.027, indexed in Pubmed: 29705220.

6. Amedro $P$, Basquin A, Gressin V, et al. Health-related quality of life of patients with pulmonary arterial hypertension associated with CHD: the multicentre cross-sectional ACHILLE study. Cardiol Young. 2016; 26(7): 1250-1259, doi: 10.1017/S1047951116000056, indexed in Pubmed: 26980152.

7. Kurzyna M, Małaczyńska-Rajpold K, Koteja A, et al. An implantable pump Lenus pro $^{\oplus}$ in the treatment of pulmonary arterial hypertension with intravenous treprostinil. BMC Pulm Med. 2017; 17(1): 162, doi: 10.1186/s12890017-0474-7, indexed in Pubmed: 29195500.

8. McKenna SP, Ratcliffe J, Meads DM, et al. Development and validation of a preference based measure derived from the Cambridge Pulmonary Hypertension Outcome Review (CAMPHOR) for use in cost utility analyses. Health Qual Life Outcomes. 2008; 6: 65, doi: 10.1186/1477-7525-6-65, indexed in Pubmed: 18718016.

9. Dębska G, Mazurek H. Validation of Polish version of CFQoL in patients with cystic fibrosis [article in Polish]. Pol Med J. 2007; 23(137): 340-343.

10. Nunnally J. Psychometric Theory, $2^{\text {nd }}$ ed. McGraw-Hill, New York 1978. 